\title{
The Dynamic Model of the Boom Portal Cranes
}

\author{
Rade Vasiljevići ${ }^{*}$, Milomir Gašić ${ }^{2}$ \\ ${ }^{1}$ High Technical School, Belgrade (Serbia) \\ ${ }^{2}$ Faculty of Mechanical and Civil Engineering, University of Kragujevac, Kraljevo (Serbia)
}

This thesis refers to an access during forming of dynamic model of a boom of portal crane. The first step defines the incentive of portal crane. In the second step simplified spatial dynamic model of a boom of portal crane has been formed and worked out. In other words, the laws of spatial oscillating of the cargo caused by the previously defined incentive have been determined. Final step defines dynamic load on the dynamic model formed.

\section{Keywords: Boom of portal crane, Dynamic model, Oscillations, Dynamic load}

\section{INTRODUCTION}

Constant development of material handling machines leads to more detailed and comprehensive analysis of both- mechanism and support structure of the machine.

The problem of oscillating of the structure is of great significance in mechanical engineering. The problem is very much present when speaking about material handling machines. In other words, modeling for the sake of dynamic analysis is particularly necessary for cranes, mining and construction machinery. For this purpose equivalent dynamic model of the machine is formed which simulates the work of actual machine. Dynamic models enable insight in dynamic work of the machines [1] and [2].

This thesis explains the access of forming of dynamic model of boom of portal crane. Before working on forming of dynamic model of boom of portal crane, several models of booms for material handling machines have been considered. Many authors have modeled the booms using different ways.

In the first place, a model of boom of coal transporter has been considered [3] and [4]. The works [3] and [4] present the boom as an independent element, which is sort of idealization since the boom is integral part of the unloading bridge.

Then, models of booms based on work of car lifters have been considered for determination of different influential parameters. The overview of these models is given in work [5].

Finally, the model of a boom of rotary crane has also been considered, which is specialized in determination of control parameters [6].

Pursuant to the completed review of models of booms of the machines, especially the models of booms in works [3] and [4], dynamic model of boom of portal crane has been formed. Therefore, dynamic model of a boom of portal crane has been formed as an independent element.

Simulation of work of the model formed can be done using different combinations of kinematic and geometrical parameters.

The focus of this thesis has been put on a boom of portal cranes, whereas the analysis of the portal, i.e. support structure of the crane remained as a subject of further research.

\section{DEFINING OF THE INCENTIVE}

The first important problem during research of dynamic behavior of portal cranes is defining the incentive.

Portal, i.e. base of the crane considered can perform only liner motion from point to point.

Kinematic motion sizes of portal crane are velocity, acceleration and distance covered. To calculate mechanisms for moving of cranes, trapezoidal speed profile are often used. Accordingly, this thesis adopts the idea that during one course the crane moves with the trapezoidal profile speed along the crane rail. A diagram which presents the adopted profile of speed is given on picture 1 , which shows diagram of motion of portal of the crane in an arbitrary time.

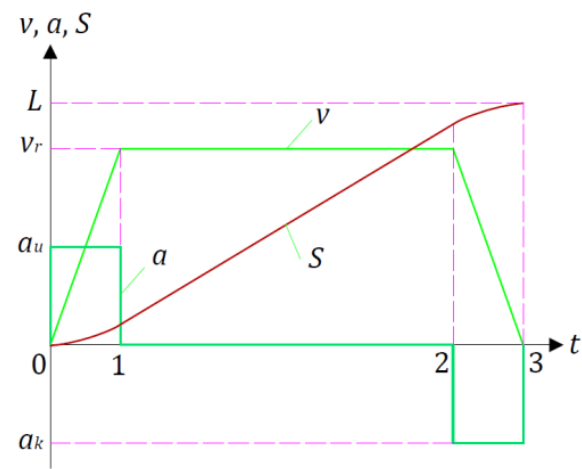

Figure 1: Time kinematic diagrams of movement of portal crane

Figure 1 shows that motion of portal is consisted out of three phases. In the phase $0-1$ (time $t_{u}$ ) portal accelerates constantly using the acceleration $a_{u}$ so the speed linearly increases and at the end of phase one it reaches speed $v_{r}$. The second phase 1-2 is the phase of balanced movement of portal during the time $t_{r}$, which is characterized by constant velocity $v_{r}$. In the phase 2-3 (time $t_{k}$ ) portal slows down constantly with deceleration $a_{k}$ until it stops. The distance covered is determined according the velocity profile, figure 1 . 


\section{DYNAMIC MODEL OF A BOOM}

\subsection{Description of model}

Boom is an integral part of portal crane. In the system of portal crane, the arrow is idealized as an individual subsystem. Regarding this, Figure 2 shows the equivalent dynamic model of the boom portal crane.

The model is designed for the determination of dynamic loads. Boom is seen as an individual subsystem that performs oscillation. Boom subsystem consists of a supporting structure of the boom and the cable system. Equivalent dynamic model is formed so that, on the one hand, keeping the main dynamic characteristics of arm and on the other hand, defined the problem is mathematically solvable.

Discretization of supporting structure of the boom is done on a light rod with a reduced weight on top.

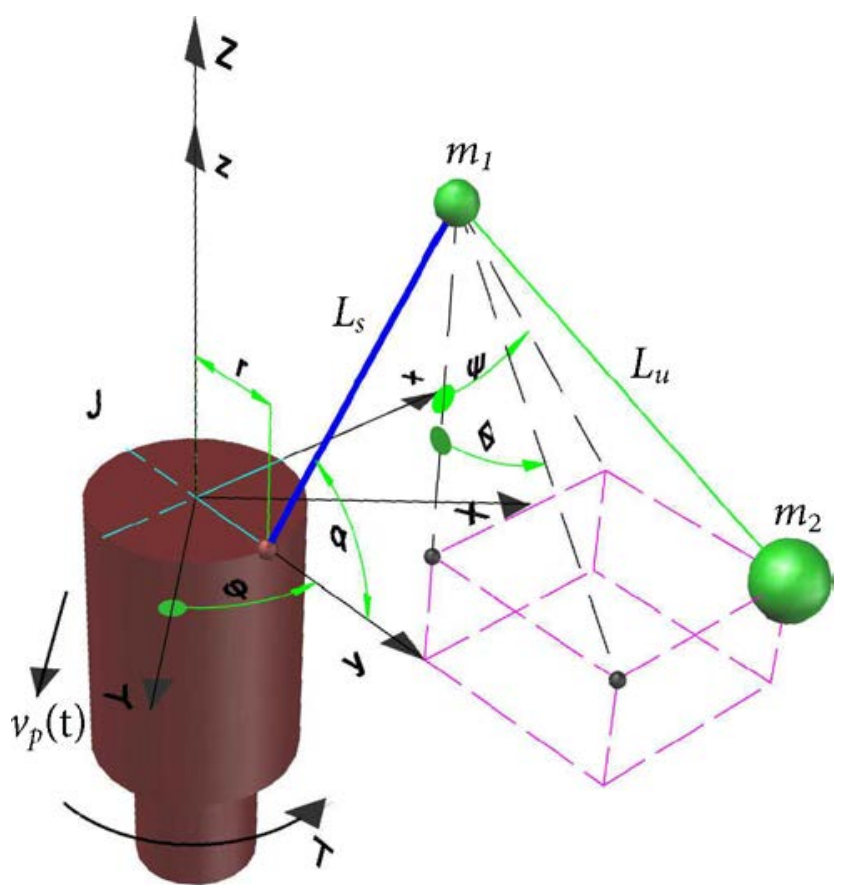

Figure 2: Dynamic model of the boom

From Figure 2 shows that the subsystem of the boom with load represented by two mass element, two light rod and a circular drive. The supporting structure of the boom was presented with a light cane which length is $L s$ and mass $m_{1}$, which is reduced to the arrowhead. Rope system stream is introduced by non flexible light cane which length is $L_{u}$ and mass $m_{2}$, enabling the oscillation of load. Mass $m_{2}$ represents the mass of the load. So arrowhead or $m_{1}$ and $m_{2}$ are connected non flexible light cane length $L_{u}$. Rotary column is represented by a circular disc of the axial moment of inertia $J$ and torque of rotation $T$. Distance joint radius of the column axis is equal to $r$.

Reduced mass $m_{1}$ is based on the recommendations in the paper [4] can be determined according to the following relation:

$$
m_{1}=\left(\frac{1}{4} \ldots \frac{1}{3}\right) m_{s}
$$

where $m_{\mathrm{s}}$ weight of of the boom.

\subsection{Mathematical formulation}

To set the mathematical formulation of a dynamic model of the boom i formed will be used Langranževe equations of the second kind. The equations of motion of elements of the boom are set on the basis of eqvivalent dynamic model shown in Figure 2. as follows:

The dynamic equations of motion of the system are

$$
\begin{gathered}
\ddot{\theta}(t)+\omega^{2} \sin \theta(t)=-\frac{1}{L_{u}} \frac{d^{2} Y_{p}}{d t^{2}} \cos \varphi \\
\ddot{\psi}(t)+\omega^{2} \sin \psi(t)=-\frac{1}{L_{u}} \frac{d^{2} Y_{p}}{d t^{2}} \sin \varphi \\
J \ddot{\varphi}(t)=T
\end{gathered}
$$

where $\theta$ angle of oscillation of the cargo in the longitudinal direction, $\psi$ angle of oscillation of cargo in the lateral direction, rotation angle, $\varphi$ rotation angle column boom, $Y_{p}$ linear displacement portal and $\omega$ angular frequency of oscillation of the load.

\subsubsection{Oscillation of cargo}

The first two equations (2a) and (2b) shall be determined by the laws of undamped oscillation of the pendulum as a function of time by generalized coordinates $\theta$ i $\psi$ due to the acceleration of the portal given diagram according to Figure 1.

In the first step, as it is considered the movement of cargo or mass $m_{2}$ in the longitudinal direction by generalized coordinate $\theta$, is observed as the mass is separated from the system, Figure 3.

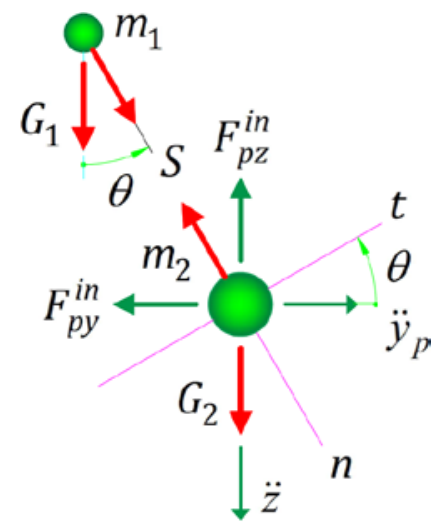

Figure 3: Oscillation of cargo in the longitudinal direction

For the purpose of determining the law swaying loads, defined differential equations of relative motion of particle or mass $m_{2}$ :

$$
m_{2} \vec{a}_{r}=\vec{F}_{p X}^{i n}+\vec{F}_{p Y}^{i n}+\overrightarrow{\mathrm{G}}_{2}+\vec{S}
$$

Equation (3) projected on the axis $t$ i $n$ natural tin inside a trihedral angle which is linked to the mass $m_{2}$.

Details of getting the law of oscillation in the longitudinal direction are previded. As there is an analogy between the oscillation burden in these two directions, the lateral direction will be given only to the final shape.

In the first step, the law of oscillation of cargo in the longitudinal direction taking shape:

$$
\ddot{\theta}(t)+\frac{g}{L_{u}} \sin \theta(t)=-\frac{1}{L_{u}} \frac{d^{2} Y_{p}}{d t^{2}} \cos \theta(t) \cos \varphi
$$

In the equation (4) ratio $g / L_{u}$ represents the circular frequency of cargo or a pendulum $\omega$. 
In the case of small oscillations can be introduced approximation that the angle $\theta$ mali, small, so $\sin \theta \approx \theta$ and $\cos \theta \approx 1$ [7]. Also, it introduces a shift $a=a_{p}=d^{2} Y_{p} / d t^{2}$. Pursuant to introduced approximations and shifts the equation (4) is linearized so it gets the following form:

$$
\ddot{\theta}(t)+\omega^{2}(t)=-\frac{1}{L_{u}} a(t) \cos \varphi
$$

Equation (5) is preferably solved by using the Laplace transform. In the first step, applying the Laplace transform the given equation is obtained:

$$
L\{(s)\}=-\frac{1}{L_{u}} L\left\{\frac{a(s)}{\left(s^{2}+\omega^{2}\right)}\right\} \cos \varphi
$$

From the kinematic diagram of Figure 1, is set aside specifically for the acceleration diagram of the need to solve the equation (6). This diagram is shown in Figure 4. It has been adopted that $a_{u}=a_{k}=h$ i $t_{u}=t_{k}=\tau$.

According to [8] for previous calculations may be adopted that $0,5\left(t_{u}+t_{k}\right)=3 \ldots 5 \mathrm{~s}$.

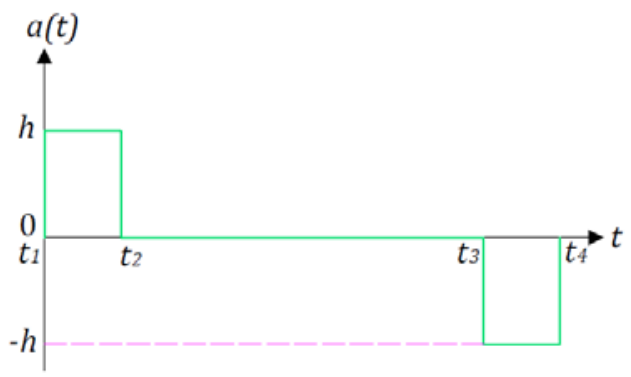

Figure 4: Diagram acceleration

Now, according to the diagram changes in acceleration time, Figure 4, the expression for acceleration in the Laplace domain can be represented in the following form:

$$
a(s)=\frac{h}{s}\left(1-e^{t_{2} s}-e^{t_{3} s}+e^{t_{4} s}\right)
$$

where $h$ input amplitude of the acceleration and $t_{i}$ corresponding time step on the diagram of acceleration.

The expression (7) becomes:

$$
L\{\theta(s)\}=\frac{h}{L_{u}} L\left\{\frac{1-e^{t_{2} s}-e^{t_{3} s}+e^{t_{4} s}}{s\left(s^{2}+\omega^{2}\right)}\right\} \cos \varphi
$$

Finally, the law of oscillation of cargo in the longitudinal direction is obtained by the expression for $\theta(s)$ is transformed into the time domain by using inverse Laplace transformations:

$$
\begin{aligned}
& \theta(t)=-\frac{h}{L_{u} \omega^{2}}\{(1-\cos \omega t)- \\
& {\left[1-\cos \omega\left(t-t_{2}\right)\right] \text { HevisideTheta }\left(t-t_{2}\right)-} \\
& {\left[1-\cos \omega\left(t-t_{3}\right)\right] \text { HevisideTheta }\left(t-t_{3}\right)+} \\
& \left.\left[1-\cos \omega\left(t-t_{4}\right)\right] \text { HevisideTheta }\left(t-t_{4}\right)\right\} \cos \varphi
\end{aligned}
$$

Analogously, we get the law of oscillation in the lateral direction by generalized coordinate $\psi$ :

$$
\begin{aligned}
& \psi(t)=-\frac{h}{L_{u} \omega^{2}}\{(1-\cos \omega t)- \\
& {\left[1-\cos \omega\left(t-t_{2}\right)\right] \text { HevisideTheta }\left(t-t_{2}\right)-} \\
& {\left[1-\cos \omega\left(t-t_{3}\right)\right] \text { HevisideTheta }\left(t-t_{3}\right)+} \\
& \left.\left[1-\cos \omega\left(t-t_{4}\right)\right] \text { HevisideTheta }\left(t-t_{4}\right)\right\} \sin \varphi
\end{aligned}
$$

The resulting laws oscillation of cargo (9) and (10) are Hevisideis (stepped) function with a linear polynomial.

Instability and capsizing of cranes contributes most swinging of cargo and when the angle of oscillation of cargo reaches its highest point. This case occurs when the pulses are time budget to each other in phase. In this case, the oscillation of cargo caused by each step acceleration constructively added and produces the highest amplitude of oscillation of the load.

The maximum amplitude of oscillation of cargo can be determined from the dynamic equations of motion of cargo in the time frame.

Equation (9) and (10) show that the maximum angle of oscillation occurs when the following conditions are met:

- each of the members within the cosine of the expression (9) and (10) is in the phase,

- multiplication of cosine members within the expression (9) and (10) by correspoding function of HevisideTheta results in 1 (it is, $t \geq t_{4}$, needed that working time is long enough so the acceleration meets the one from diagram).

Cosine members within the expression (9) and (10) are at the stage when the four steps of a diagram acceleration are performed by in constructively summed up perfect perid of time. In the worst case, the angle of oscillation is growing four times the oscillation caused by a single step input acceleration. The maximum size of the angle of oscillation of cargo in the longitudinal and lateral direction is equal to:

$$
\left|\theta_{\max }\right|=\left|\psi_{\max }\right|=4 \frac{h}{g}
$$

To produce a maximum of swinging of cargo it is necessary that the steps of acceleration are totaly within phase limits, ie, it is necessary that two conditions are met.

The first condition requires that the time interval between the first and second steps, as well as the third and fourth must be half the oscillatory period $\tau$, that is mathematically written:

$$
\begin{aligned}
& t_{2}-t_{1}=t_{2}=(0,5+n) \tau, \mathrm{i} \\
& t_{4}-t_{3}=(0,5+n) \tau
\end{aligned}
$$

where $n=1,2,3 \ldots$

A time delay is necessary because the steps in sets have different marks. Therefore, the time delay is equivalent to causing retention phase $\pi$ to the opposite function signs, which all finally canceled the change phase and leads two functions in phase.

The second condition requires that the time interval between the second and third pulses must contain multiple retention time period $\tau$, that is mathematically written:

$$
t_{3}-t_{2}=n \tau
$$

where $n=1,2,3$..

In other words, this is true because the second and third step have the same sign and phase change of $2 \pi$ claims that there are in phase.

\subsubsection{Dynamic load}

In accordance with the adopted generalized coordinates of oscillation of the dynamic model of boom, dynamic bending moment occurs due to oscillation loads in two directions: 
- dynamic bending moment due to the oscillation of cargo in the longitudinal direction, and

- dynamic bending moment due to oscillation of cargo in the lateral direction.

Dynamic bending moment due to the oscillation of the cargo in the longitudinal direction reads, figure 2:

$$
\begin{aligned}
& M_{d i n, p}=m_{1} g\left(L_{s} \cos \alpha+r\right)+ \\
& m_{2}\left(g+L_{u} \dot{\theta}^{2} \cos \theta\right)\left(L_{u} \sin \theta+L_{s} \cos \alpha+r\right)+ \\
& m_{2} L_{u} \dot{\theta}^{2} \sin \theta\left(L_{s} \sin \alpha-L_{u} \cos \theta\right)
\end{aligned}
$$

Dynamic bending moment due to oscillation loads in the lateral direction can be twofold, slika 2:

$$
\begin{aligned}
& M_{d i n, b 1}=m_{2}\left(g+L_{u} \dot{\psi}^{2} \cos \psi\right) L_{u} \sin \psi+ \\
& m_{2} L_{u} \dot{\psi}^{2} \sin \psi\left(L_{s} \sin \alpha-L_{u} \cos \psi\right) \\
& M_{d i n, b 2}=m_{2} L_{u} \dot{\psi}^{2} \cos \psi\left(L_{s} \cos \alpha+r\right)
\end{aligned}
$$

\section{NUMEROUS EXAMPLES}

In this subchapter shall in the case of portal cranes applied theoretical dynamic analysis laid out in subheadings 2 and 3 to verify its correctness and the formation of conclusions.

Numerous example is done for the portal crane, which are known following baseline data [9]: $v_{p}=0,6 \mathrm{~m} / \mathrm{s}$, $m_{t}=10000 \mathrm{~kg}, m_{s}=9200 \mathrm{~kg}, r=1 \mathrm{~m}, L_{s}=30 \mathrm{~m}$ and $\alpha=30^{\circ}$.

Results and discussion of the results of numerical examples are given in the following sub-headings.

\subsection{Results of the analysis of oscillations of cargo}

Movement of portal on rails is described diagram speed $v_{p}(\mathrm{t})$. Figure 5 shows the three profiles speed portal.

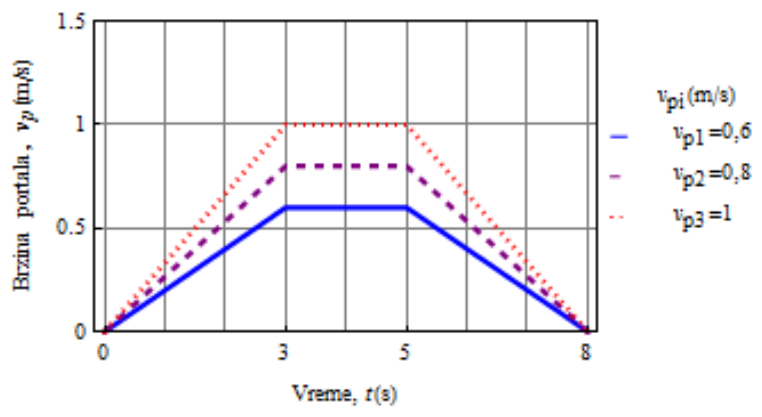

Figure 5: Profiles speed portal $v_{p}(t)$

Changing the angle of oscillation of cargo in the longitudinal direction $\theta$ speed $v_{p 1}=0,6 \mathrm{~m} / \mathrm{s}$ and $L_{u}=1 \mathrm{~m}$, based on the expression (9), shown in Figure 6.

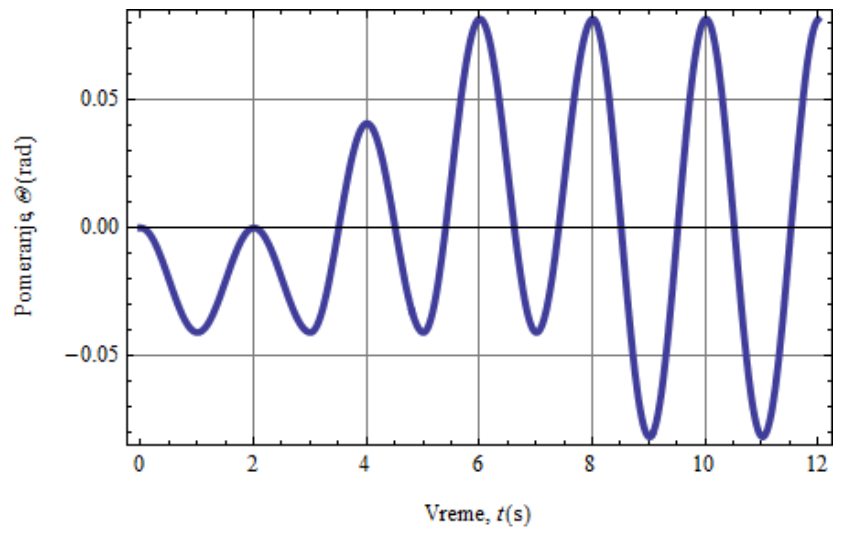

Figure 6: Changing the angle of oscillation of cargo in the longitudinal direction of $v_{1}=0,6 \mathrm{~m} / \mathrm{s}$
Changing the angle of oscillation of cargo in the lateral direction $\psi$ is exactly the same change in the angle of oscillation of the cargo in the longitudinal direction $\theta$.

\subsubsection{The impact velocity and acceleration of the portal}

The impact velocity and acceleration of the movable portal can be viewed through consideration of oscillation of cargo due to the implementation of the profile velocity portal $v_{p 3}$, which represent the extreme performance portal crane.

In Figure 7, based on the expression (9) shows the angle of oscillation of the cargo in the longitudinal direction for different speeds of the portal.

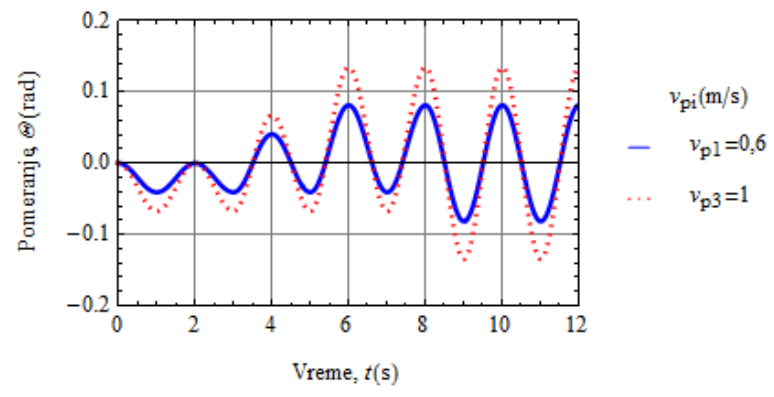

Figure 7: The angle of oscillation of cargo in the long. direction for different speeds of the portal $v_{p}(t)$

Figure 7 shows that the angle of oscillation of cargo increases with increasing speed of the portal.

\subsubsection{Influence of length of cable system}

Table 1 shows the circular frequencies of cargo depending on the length of the cable system. Table 1 shows that the increase in length of cable system reduces the angular frequency of oscillation of the load.

Table 1: Circular frequencies of oscillation cargo

\begin{tabular}{|c|c|}
\hline $\begin{array}{l}\text { Lengths of cable } \\
\text { system, } L_{u}[\mathrm{~m}]\end{array}$ & $\begin{array}{l}\text { Circular } \\
\text { frequency, } \omega\left[\mathrm{s}^{-1}\right]\end{array}$ \\
\hline 1 & 3,14 \\
\hline 3 & 1,81 \\
\hline 5 & 1,40 \\
\hline 7 & 1,18 \\
\hline 9 & 1,04 \\
\hline
\end{tabular}

Again, influence of the length of the rope can be viewed through comparative investigation of oscillation load for two different lengths of cable system $L_{u}=1 \mathrm{~m}$ i $L_{u}=5 \mathrm{~m}$. This size has an impact on the change of oscillation period.

In Figure 8, on the basis of the expression (9), shown is the angle of oscillation of the cargo in the longitudinal direction for two different lengths of the cable system.

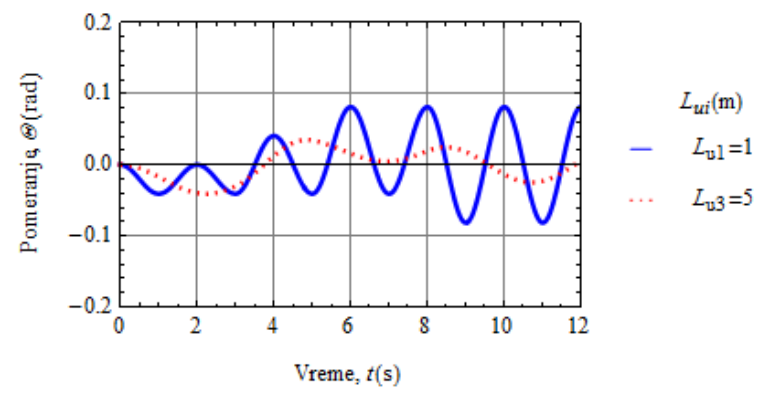

Figure 8: The oscilating angle of the load in longitudinal direction for diferentlengths of cable system $L_{u}$ 
Figure 8 shows the angle of oscillation of cargo decreases with increasing length of the cable system. Also, there is an increase oscillation period.

\subsection{Results of the analysis of dynamic loads}

Dynamic changes of the bending moment due to oscillation of cargo in the longitudinal direction $\theta$, on the basis of the expression (14), is shown in Figure 9.

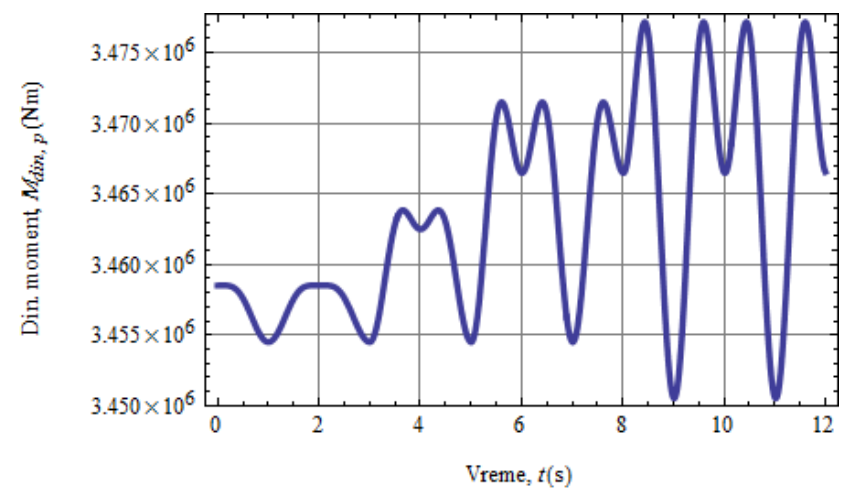

Figure 9: Changing the dynamic bending moment due to the oscillation of the cargo in the longitudinal direction

Dynamic changes of the bending moment due to oscillation of cargo in the lateral direction $\psi$, on the basis of the expression (15) and (16), is shown in Figure 10 and 11.

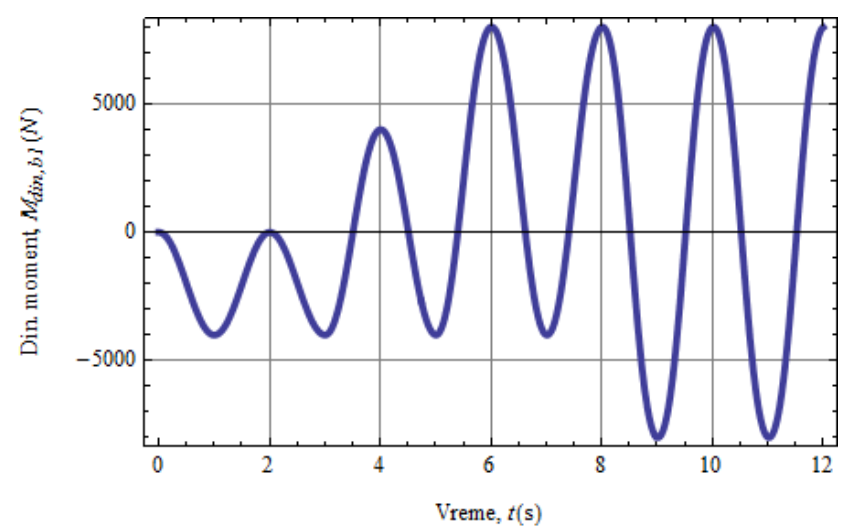

Figure 10: Changing the dynamic bending moment due to oscillation loads in the lateral direction $-b_{1}$

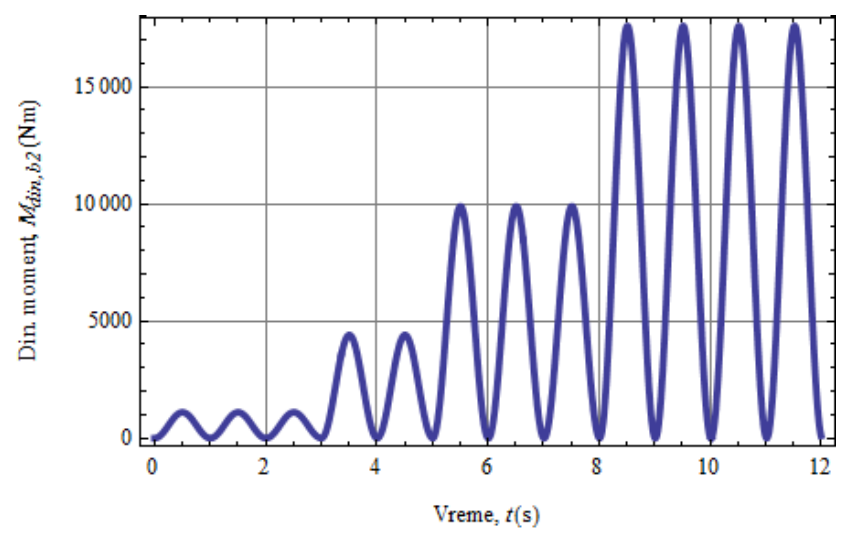

Figure 11: Changing the dynamic bending moment due to oscillation loads in the lateral direction $-b_{2}$

4.2.1. The impact velocity and acceleration of the portal

The impact velocity and acceleration of the movable portal can be viewed through consideration of dynamic loads due to implementation profile velocity portal $v_{p 3}$, which represent the extreme performance portal crane.
Figures 12...14, on the basis of the expression (14), (15) and (16), are considered dynamic bending moments for different speeds of the portal. This figures shows that the dynamic bending moments increase with the speed of the portal.

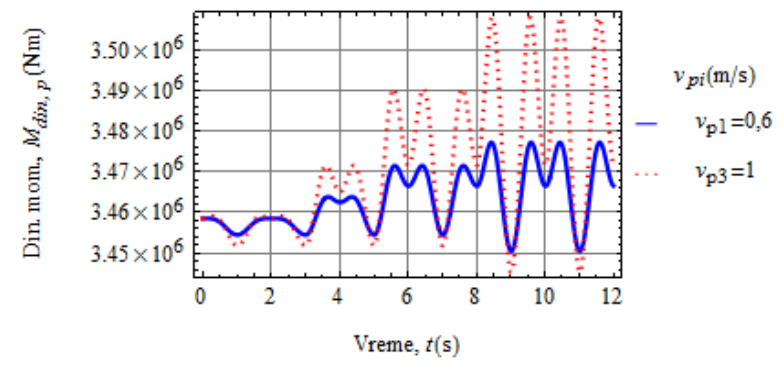

Figure 12: Changing the dynamic bending moment, for different speeds, due to oscillation of cargo in the longitudinal direction

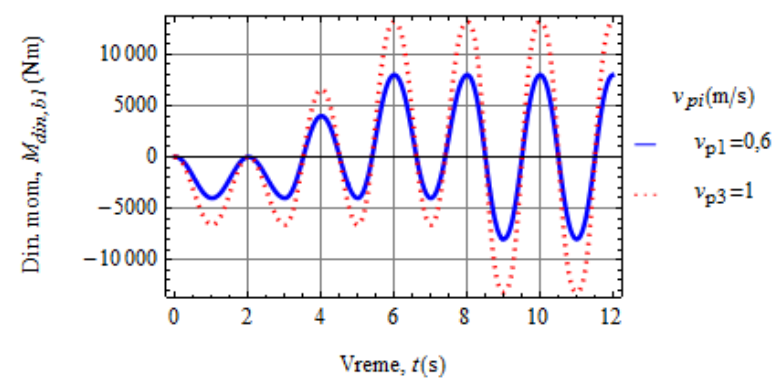

Figure 13: Changing the dynamic bending moment, for different speeds, due to oscillation of cargo in the lateral direction $-b_{1}$

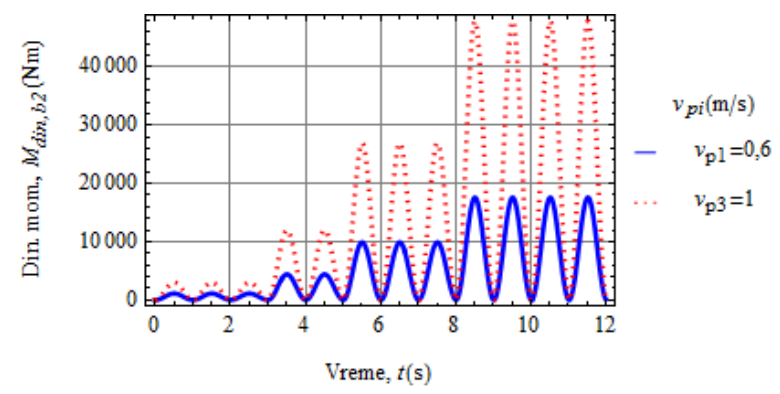

Figure 14: Changing the dynamic bending moment, for different speeds, due to oscillation of cargo in the lateral direction $-b_{2}$

The figures shown 12...14 show that the dynamic moments increase with increasing speed of the portal.

\subsubsection{The influence of the length of the cable system}

In figure 15, 16 and 17, on the basis of expressions (15), (16) and (17), are shown the dynamic bending moments in longitudinal and lateral direction for three different lengths of the cable system. These images show that the first two dynamic bending moment increase with the length of the cable system, while the third is reduced. 


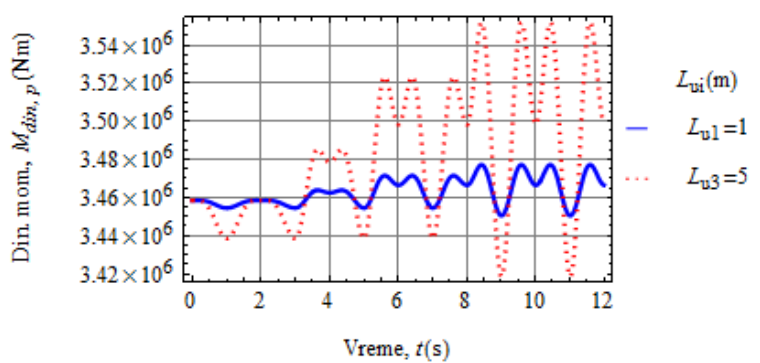

Figure 15: Changing the dynamic bending moment for different lengths of the cable system, due to oscillation of cargo in the longitudinal direction

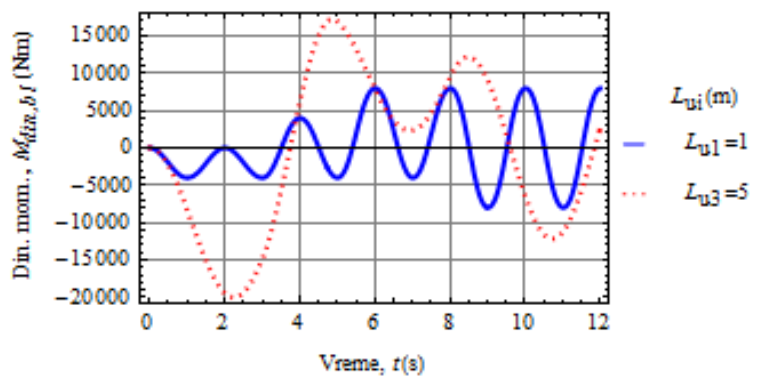

Figure 16: Changing the dynamic bending moment for different lengths of the cable system, due to oscillation of cargo in the lateral direction $-b_{1}$

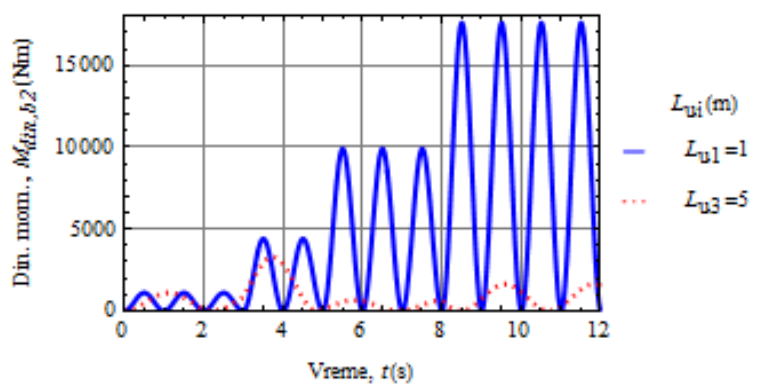

Figure 17: Changing the dynamic bending moment for different lengths of the cable system, due to oscillation of cargo in the lateral direction - $b_{2}$

\section{CONCLUSION}

In this paper, a simplified mathematical model of the boom portal cranes. Is presented dynamic model gives the final equation of oscillation in the of cargo area in two mutually perpendicular directions (longitudinal and lateral), in the overall numbers. This feature of dynamic analytical model makes it universal, so it can be applied to the portal cranes of various performance, and the like cranes or hoists that have similar of the boom.

Based on the formed dynamic model has been shown that the laws of oscillation loads in two perpendicular directions describe Hevisideis (stepped) functions with a linear polynomial.

In the particular example of of the boom portal to rotary the crane is scheduled as follows:
- influence speed at the size of the decline of cargo,

- changes oscillating loads in two mutually perpendicular directions (longitudinal and lateral),

- impact of changes in the length of the rope to the size of the decline of cargo,

- changes in dynamic loads due to oscillation of cargo in two perpendicular directions (longitudinal and lateral).

The results of dynamic models confirm its validity.

Further, the dynamic model is the basis for the research of influential parameters on the dynamic behavior of portal crane high performance.

\section{REFERENCES}

[1] D. Ostrić, "Dynamics of bridge cranes”, Mechanical Engineering, Belgrade (Serbia), (1988)

[2] M. Georgijević, "Dynamics crane - experimental and model analysis", Andrejević Foundation, Belgrade (Serbia), (1996)

[3] V. Gašić, "Dynamic behaviour identification of bridge type stacker - reclaimer with bucket chain booms in power plants”, M.Sc Thesis, University of Belgrade (Serbia), (2004)

[4] Z. Petković, V. Gašić and S. Bošnjak, "Comparative Overview Of Simplified Dynamic And Finite Element Model Of Boom Structure At Special Coal Stacker-Reclaimer", Journal of Mechanical Engineering Desing, Vol. 8(2), pp. 41-46, (2005)

[5] M. Savković, "Contribution To Modelling Of Autocrane Boom”, IMK-14 - Research \& Development in Heavy Machinery, Vol. 9(1/2), pp. 123-128, (2003)

[6] T. Gustafsson, "On the Design and Implementation of a Rotary Crane Controller", European Journal of Control”, Appl. Mech. Rev, Vol. 2(3), pp. 166-175, (1996)

[7] LJ. Radosavljević, “Theory of oscillations”, Mechanical Engineering, Belgrade (Serbia), (1981)

[8] D. Ostrić, S. Tošić, “Crane”, Mechanical Engineering, Belgrade (Serbia), (2005)

[9] А. Ланг, И. Мазовер, В. Майзель, “Портальные краны - расчет и конструирование”, Государственное научно-техническое издательство машинстриельной литературы, Москва-Ленинград (Руссиа), (1962) 